\title{
ANALYTIC AND GEOMETRIC PROPERTIES OF GENERIC RICCI SOLITONS
}

\author{
G. Catino $^{1}$, P. Mastrolia ${ }^{2}$, D. D. Monticelli ${ }^{3}$ and M. Rigoli ${ }^{4}$,
}

\begin{abstract}
The aim of this paper is to prove some classification results for generic shrinking Ricci solitons. In particular, we show that every three dimensional generic shrinking Ricci soliton is given by quotients of either $\mathbb{S}^{3}, \mathbb{R} \times \mathbb{S}^{2}$ or $\mathbb{R}^{3}$, under some very weak conditions on the vector field $X$ generating the soliton structure. In doing so we introduce analytical tools that could be useful in other settings; for instance we prove that the Omori-Yau maximum principle holds for the $X$-Laplacian on every generic Ricci soliton, without any assumption on $X$.
\end{abstract}

\section{INTRODUCTION}

The fundamental problem of capturing the topological properties of a manifold by its metric structure opened, in the last decades, extremely fruitful areas of mathematics. From this perspective, there has been an increasing interest in the study of Riemannian manifolds endowed with metrics satisfying special structural equations, possibly involving curvatures and vector fields. One of the most important example is represented by Ricci solitons, that have become the subject of a rapidly increasing investigation since the appearance of the seminal works of R. Hamilton, [10], and G. Perelman, [17]. We recall that if $(M, g)$ is a $m$-dimensional, connected, Riemannian manifold with metric $g$, a soliton structure $(M, g, X)$ on $M$ is the choice (if any) of a smooth vector field $X$ on $M$ and a constant $\lambda \in \mathbb{R}$ such that

$$
\operatorname{Ric}+\frac{1}{2} \mathcal{L}_{X} g=\lambda g
$$

where Ric denotes the Ricci tensor of the metric $g$ and $\mathcal{L}_{X} g$ is the Lie derivative of the metric in the direction of $X$ : the constant $\lambda$ is sometimes called the soliton constant. The soliton is expanding, steady or shrinking if, respectively, $\lambda<0, \lambda=0$ or $\lambda>0$. If $X$ is the gradient of a potential $f \in C^{\infty}(M)$ the soliton is called a gradient Ricci soliton and (1.1) becomes

$$
\operatorname{Ric}+\operatorname{Hess}(f)=\lambda g .
$$

In this case, using the symmetry of the tensor Hess $(f),(1.2)$ and the second Bianchi identity, one proves the validity of the fundamental equation

$$
\frac{1}{2} \nabla S=\operatorname{Ric}(\nabla f,)^{\sharp},
$$

where $S$ is the scalar curvature and $\sharp: T^{*} M \rightarrow T M$ is the musical isomorphism. Equation (1.3), together with Hamilton identity (see e.g. [10])

$$
S+|\nabla f|^{2}-2 \lambda f=C, \quad C \in \mathbb{R},
$$

is responsible for a number of basic properties related to the geometry of gradient solitons. For instance, from (1.4) one deduces an upper bound on the growth of the weighted volume $\operatorname{vol}_{f}\left(B_{r}\right)=\int_{B_{r}} e^{-f} d \mu$ of

Date: June 6, 2018.

2010 Mathematics Subject Classification. 53C20, 53C25.

Key words and phrases. Ricci solitons, Omori-Yau maximum principle, Rigidity results.

${ }^{1}$ Politecnico di Milano, Italy. Email: giovanni.catino@polimi.it. Supported by GNAMPA projects "Equazioni differenziali con invarianze in analisi globale" and "Equazioni di evoluzione geometriche e strutture di tipo Einstein".

${ }^{2}$ Università degli Studi di Milano, Italy. Email: paolo.mastrolia@gmail.com. Partially supported by FSE, Regione Lombardia.

${ }^{3}$ Università degli Studi di Milano, Italy. Email: dario.monticelli@gmail.com. Supported by GNAMPA projects "Equazioni differenziali con invarianze in analisi globale" and "Analisi Globale ed Operatori Degeneri".

${ }^{4}$ Università degli Studi di Milano, Italy. Email: marco.rigoli@unimi.it.

The first, the second and the third authors are members of the Gruppo Nazionale per l'Analisi Matematica, la Probabilità e le loro Applicazioni (GNAMPA) of the Istituto Nazionale di Alta Matematica (INdAM). 
geodesic balls in $M$ (see [4]). For generic Ricci solitons $(M, g, X)$, that is, when $X$ is not the gradient of a potential, neither (1.3) nor Hamilton's identity (1.4) are available. In case of (1.3) this is technically due to the fact that, in the generic setting, the symmetry of $\operatorname{Hess}(f)$, i.e.

$$
\operatorname{Hess}(f)(Y, Z)=\operatorname{Hess}(f)(Z, Y)
$$

for every smooth vector fields $Y$ and $Z$, is replaced by the much more involved "commutation rule"

$$
g\left(\nabla_{Y} X, Z\right)=2 \lambda g(Y, Z)-2 \operatorname{Ric}(Y, Z)-g\left(\nabla_{Z} X, Y\right) .
$$

Nevertheless, even in in this more general situation, some important equations valid for gradient solitons still hold, basically in the same form (see formulas (2.3) and (2.4) in Lemma 2.1 below). These equations have been recently considered in [13] to infer some upper and lower estimates on $\inf _{M} S$ and $\sup _{M}|T|$, where $T$ is the traceless Ricci tensor, under a growth condition on $|X|$. Using Lemma 2.1 below we free $X$ from this restriction, improving on our Theorems 1.1 and 1.2 of [13]; for instance, Theorem 1.1 just quoted becomes

Theorem 1.1. Let $(M, g, X)$ be a complete, generic Ricci soliton of dimension $m$ and scalar curvature S. Set $S_{*}=\inf _{M} S$.

i) If $\lambda<0$ then $m \lambda \leq S_{*} \leq 0$. Furthermore, if $S\left(x_{0}\right)=S_{*}=m \lambda$ for some $x_{0} \in M$, then $(M, g)$ is Einstein and $X$ is a Killing field; on the other hand, if $S\left(x_{0}\right)=S_{*}=0$ for some $x_{0} \in M$, then $(M, g)$ is Ricci flat and $X$ is a homothetic vector field.

ii) If $\lambda=0$ then $S_{*}=0$. Furthermore, if $S\left(x_{0}\right)=S_{*}=0$ for some $x_{0} \in M$, then $(M, g)$ is Ricci flat and $X$ is a Killing field.

iii) If $\lambda>0$ then $0 \leq S_{*} \leq m \lambda$. Furthermore, if $S\left(x_{0}\right)=S_{*}=0$ for some $x_{0} \in M$, then $(M, g)$ is flat and $X$ is a homethetic vector field, while $S_{*}<m \lambda$ unless $M$ is compact, Einstein and $X$ is a Killing field.

Note that, in the original statement of [13], in case iii) we concluded that if $S\left(x_{0}\right)=S_{*}=0$ then $(M, g)$ is Ricci flat; the present stronger conclusion is then obtained by applying Theorem 4.1 of [22]. As a matter of fact, part iii) of Theorem 1.1 and the differential inequality (2.20) below are key steps in the proof of the following classification results for generic shrinking solitons. To fix the notation, we let $o$ be some chosen origin in $M$ and we set $r(x)=\operatorname{dist}_{(M, g)}(x, o)$.

Theorem 1.2. Let $(M, g, X)$ be a complete generic shrinking Ricci soliton of dimension three. Furthermore, if $M$ is noncompact, assume that the scalar curvature is bounded and $|\nabla X|=o(|X|)$ as $r \rightarrow \infty$. Then $(M, g)$ is isometric to a finite quotient of either $\mathbb{S}^{3}, \mathbb{R} \times \mathbb{S}^{2}$ or $\mathbb{R}^{3}$.

In higher dimensions Theorem 1.2 generalizes to

Theorem 1.3. Let $(M, g, X)$ be a complete generic shrinking Ricci soliton of dimension $m>3$. Furthermore, if $M$ is noncompact, assume that the scalar curvature is bounded and $|\nabla X|=o(|X|)$ as $r \rightarrow \infty$. If, for some $\Lambda>0, \mid$ Ric $\mid \leq \Lambda S$ and

$$
|W| S \leq \sqrt{\frac{2(m-1)}{m-2}}\left(|T|-\frac{1}{\sqrt{m(m-1)}} S\right)^{2},
$$

then $(M, g)$ is isometric to a finite quotient of either $\mathbb{S}^{m}, \mathbb{R} \times \mathbb{S}^{m-1}$ or $\mathbb{R}^{m}$.

Theorems 1.2 and 1.3 extend to the non-gradient case the previous results of Perelman [16], Cao et al. [3] and the first author [5] and provide results in the non conformally flat case, which was treated by the first author et al. [6].

Remark 1.4. Tracing equation (1.1) it follows that the previous theorems in particular hold simply assuming that $|\nabla X|$ is bounded and, if $m>3$, inequality (1.5). 
In proving Theorems 1.1, 1.2 and 1.3 we shall need certain geometric and analytic preliminary results that are interesting in their own, which we state and prove in Sections 2 and 3. In particular, in Section 2 (see Proposition 2.3) we show that the Omori-Yau maximum principle, and therefore the weak maximum principle, holds for the $X$-Laplacian (defined below in equation (2.1)) on every generic Ricci soliton, without any assumption on $X$; this extends the previous result in [9] to the non-gradient setting. In Section 3 we give a sufficient condition for the parabolicity of a large class of linear second order operators, including the $X$-Laplacian. Finally, in Section 5 we give a classification result for complete generic Ricci solitons with constant scalar curvature (see Theorem 5.4).

\section{Geometry of a generic Ricci soliton}

On a generic Ricci soliton structure $(M, g, X)$ we introduce (see for instance [13]) the differential operator $\Delta_{X}$, that we call the $X$-Laplacian and that acts on a function $u \in \operatorname{Lip}_{l o c}(M)$ as

$$
\Delta_{X} u=\Delta u-g(X, \nabla u) .
$$

Of course, (2.1) has to be understood in the weak sense. Note that in case $X=\nabla f$, that is, in case of a gradient Ricci soliton with potential $f$, the operator $\Delta_{X}$ coincides with the symmetric diffusion operator (sometimes called $f$-Laplacian or drifted Laplacian)

$$
\Delta_{f} u=\Delta u-g(\nabla f, \nabla u)=e^{f} \operatorname{div}\left(e^{-f} \nabla u\right) .
$$

We recall that the Omori-Yau maximum principle for $\Delta_{X}$ holds on $(M, g)$ if, given any function $u \in$ $C^{2}(M)$ with $u^{*}=\sup _{M} u<+\infty$, there exists a sequence $\left\{z_{k}\right\}_{k} \subset M$ such that

$$
\text { (i) } u\left(z_{k}\right)>u^{*}-\frac{1}{k}, \quad(\text { ii })\left|\nabla u\left(z_{k}\right)\right|<\frac{1}{k}, \quad \text { (iii) } \Delta_{X} u\left(z_{k}\right)<\frac{1}{k}
$$

for each $k \in \mathbb{N}$. We also say that the weak maximum principle hold for $\Delta_{X}$ if only $(i)$ and $(i i i)$ in $(2.2)$ are met.

From now on we shall freely use the notation of the method of the moving frame referring to a fixed local orthonormal coframe for computations. We fix the index convention $1 \leq i, j, k, s, \ldots \leq m=\operatorname{dim} M$, we let $T=\operatorname{Ric}-\frac{S}{m} g$ denote the traceless Ricci tensor and we set $T_{i j}, R_{i j k s}$ and $W_{i j k s}$ to denote the components of $T$, of the Riemann and of the Weyl curvature tensors respectively. Further notation will be clear from the context. The following equations have been obtained in Lemmas 9 and 10 of [13].

Lemma 2.1. Let $(M, g, X)$ be a generic Ricci soliton. Then

$$
\begin{aligned}
\frac{1}{2} \Delta_{X} S & =\lambda S-\mid \text { Ric }\left.\right|^{2}=\lambda S-\frac{S^{2}}{m}-|T|^{2}, \\
\frac{1}{2} \Delta_{X}|T|^{2} & =|\nabla T|^{2}+2\left(\lambda-\frac{m-2}{m(m-1)} S\right)|T|^{2}+\frac{4}{m-2} \operatorname{tr}\left(t^{3}\right)-2 T_{k i} T_{s j} W_{k s i j},
\end{aligned}
$$

where the endomorphism $t: T M \rightarrow T M$ is defined by $t(Y)=T(Y,)^{\sharp}$ and $\operatorname{tr}\left(t^{3}\right)$ is the trace of the operator $t^{3}=t \circ t \circ t$.

Having defined $r(x)$ in the Introduction we now deduce an upper estimate for $\Delta_{X} r$ which depends only on a lower bound for

$$
\operatorname{Ric}_{X}=\operatorname{Ric}+\frac{1}{2} \mathcal{L}_{X} g
$$

We denote with $\operatorname{cut}(o)$ the cut-locus of the origin $o$.

Proposition 2.2. Let $(M, g)$ be a complete Riemannian manifold of dimension $m \geq 2$ and let $X$ be a vector field on $M$. Suppose that, for some $F \in C^{0}\left(\mathbb{R}_{0}^{+}\right)$,

$$
\operatorname{Ric}_{X} \geq-(m-1) F(r) g \text {. }
$$


Then there exist a constant $C>0$ and $\delta>0$ sufficiently small such that

$$
\Delta_{X} r(x) \leq C+(m-1) \int_{\delta}^{r(x)} F(t) d t
$$

pointwise on $M \backslash(\{o\} \cup \operatorname{cut}(o))$ and weakly on $M$.

Proof. Fix $x \in M \backslash(\{o\} \cup \operatorname{cut}(o))$ and let $\gamma:[0, l] \rightarrow M$, with $l=\operatorname{length}(\gamma)$, be a minimizing geodesic such that $\gamma(0)=o$ and $\gamma(l)=x$. Note that $F(r(\gamma(t)))=F(t)$ for every $t \in[0, l]$. From Böchner formula, see Remark 2.6 below, applied to the distance function $r$ outside $\{o\} \cup \operatorname{cut}(o)$ we have

$$
0=|\operatorname{Hess}(r)|^{2}+\operatorname{Ric}(\nabla r, \nabla r)+\langle\nabla \Delta r, \nabla r\rangle
$$

so that, using the inequality

$$
|\operatorname{Hess}(r)|^{2} \geq \frac{(\Delta r)^{2}}{m-1}
$$

it follows that the function $\psi(t)=(\Delta r) \circ \gamma(t)$, for $t \in(0, l]$ satisfies the Riccati differential inequality

$$
\psi^{\prime}+\frac{1}{m-1} \psi^{2} \leq-\operatorname{Ric}(\dot{\gamma}, \dot{\gamma}) \quad \text { on }(0, l],
$$

where $\dot{\gamma}(t)$ is the tangent vector of $\gamma$ at time $t$. We now define $\psi_{X}(t)=\left(\Delta_{X} r\right) \circ \gamma(t)=\psi(t)-\langle X, \nabla r\rangle \circ \gamma(t)$ so that

$$
\psi_{X}^{\prime}=\psi^{\prime}-(\langle X, \nabla r\rangle \circ \gamma)^{\prime}=\psi^{\prime}-\frac{1}{2} \mathcal{L}_{X} g(\dot{\gamma}, \dot{\gamma}) .
$$

Thus using (2.8) we obtain

$$
\psi_{X}^{\prime} \leq-\frac{\psi^{2}}{m-1}-\operatorname{Ric}(\dot{\gamma}, \dot{\gamma})-\frac{1}{2} \mathcal{L}_{X} g(\dot{\gamma}, \dot{\gamma})
$$

and from $(2.5)$

$$
\psi_{X}^{\prime} \leq-\operatorname{Ric}_{X}(\dot{\gamma}, \dot{\gamma})
$$

From assumption (2.6) it follows that

$$
\psi_{X}^{\prime}(t) \leq(m-1) F(t)
$$

for $t \in(0, l]$. Choosing $\delta>0$ so small that the geodesic ball $B_{\delta}(o)$ is inside the domain of the normal coordinates at $o$ and setting $C=\max _{\partial B_{\delta}} \Delta_{X} r$, integration for $t$ between $\delta$ and $r(x)$ gives

$$
\Delta_{X} r(x) \leq C+(m-1) \int_{\delta}^{r(x)} F(t) d t
$$

pointwise in $M \backslash(\{o\} \cup \operatorname{cut}(o))$, so that (2.7) follows. To show the validity of this inequality weakly on all of $M$ we argue in a way similar to that of Lemma 2.5 of [19]; for the sake of completeness we report the reasoning. By an observation of Cheng and Yau, [8], we can consider an exhaustion $\left\{\Omega_{n}\right\}$ of $M \backslash \operatorname{cut}(o)$ by bounded domains with smooth boundaries starshaped with respect to $o$. Fix $n$ and let $\nu$ be the outward unit normal to $\partial \Omega_{n}$; denote with $\rho(x)=\operatorname{dist}\left(x, \partial \Omega_{n}\right)$ with the convention that $\rho(x)>0$ if $x \in \Omega_{n}$ and $\rho(x)<0$ if $x \notin \Omega_{n}$. Thus $\rho$ is the radial coordinate for the Fermi coordinates relative to $\partial \Omega_{n}$. By Gauss lemma $|\nabla \rho|=1$ and $\nabla \rho=-\nu$ on $\partial \Omega_{n}$. Let

$$
\Omega_{n, \varepsilon}=\left\{x \in \Omega_{n}: \rho(x)>\varepsilon\right\}
$$

for some $\varepsilon>0$ sufficiently small and define the Lipschitz function

$$
\psi_{\varepsilon}(x)=\left\{\begin{array}{lll}
1 & \text { if } & x \in \Omega_{n, \varepsilon} \\
\rho(x) / \varepsilon & \text { if } & x \in \Omega_{n} \backslash \Omega_{n, \varepsilon} \\
0 & \text { if } & x \in M \backslash \Omega_{n} .
\end{array}\right.
$$


Let $\varphi \in C_{0}^{\infty}(M), \varphi \geq 0$; then $\varphi \psi_{\varepsilon} \in W_{0}^{1,2}\left(\Omega_{n}\right)$ and $\varphi \psi_{\varepsilon} \geq 0$. Because of the validity of (2.7) in $\Omega_{n} \backslash\{o\}$ and Gauss lemma, having set $G(x)$ for the right-hand side of (2.7) we have

$$
\begin{aligned}
\int_{\Omega_{n}} G(x) \varphi \psi_{\varepsilon} & \geq \int_{\Omega_{n}}-g\left(\nabla r, \nabla\left(\varphi \psi_{\varepsilon}\right)\right)-g(X, \nabla r) \varphi \psi_{\varepsilon} \\
& =-\left(\int_{\Omega_{n}} g(\nabla r, \nabla \varphi) \psi_{\varepsilon}+g(X, \nabla r) \varphi \psi_{\varepsilon}\right)-\frac{1}{\varepsilon} \int_{\Omega_{n} \backslash \Omega_{n, \varepsilon}} g(\nabla r, \nabla \rho) \varphi .
\end{aligned}
$$

Therefore, by the co-area formula,

$$
\int_{\Omega_{n}} G(x) \varphi \psi_{\varepsilon} \geq-\left(\int_{\Omega_{n}} g(\nabla r, \nabla \varphi) \psi_{\varepsilon}+g(X, \nabla r) \varphi \psi_{\varepsilon}\right)-\frac{1}{\varepsilon} \int_{0}^{\varepsilon} d t \int_{\partial \Omega_{n, t}} g(\nabla r, \nabla \rho) \varphi .
$$

Letting $\varepsilon \downarrow 0^{+}$we get

$$
\int_{\Omega_{n}} G(x) \varphi \geq-\left(\int_{\Omega_{n}} g(\nabla r, \nabla \varphi)+g(X, \nabla r) \varphi\right)+\int_{\partial \Omega_{n}} g(\nabla r, \nu) \varphi,
$$

and since $\Omega_{n}$ is starshaped,

$$
\int_{\Omega_{n}} G(x) \varphi \geq-\left(\int_{\Omega_{n}} g(\nabla r, \nabla \varphi)+g(X, \nabla r) \varphi\right) .
$$

By letting $n \rightarrow+\infty$, observing that $\operatorname{cut}(o)$ has measure 0 and $\operatorname{supp} \varphi$ is compact, using Fatou's Lemma the above yields

$$
\int_{M} G(x) \varphi \geq-\left(\int_{M} g(\nabla r, \nabla \varphi)+g(X, \nabla r) \varphi\right)
$$

giving the validity of (2.7) weakly on all of $M$.

If we let $(M, g, X)$ be a generic Ricci soliton with $M$ complete, then (2.6) holds in the form

$$
\operatorname{Ric}_{X}=\lambda g
$$

so that we can choose $F(t)=-\frac{\lambda}{m-1}$. It follows that

$$
\Delta_{X} r \leq C-\lambda(r-\delta)
$$

on $M \backslash(\{o\} \cup \operatorname{cut}(o))$ and weakly on all of $M$.

Proceeding in a way similar to that in the proof of Theorem 3 in [2] we have the following proposition that improves on [13].

Proposition 2.3. Let $(M, g, X)$ be a generic Ricci soliton with $(M, g)$ complete. Then the Omori-Yau and therefore the weak maximum principles hold for the operator $\Delta_{X}$.

We explicitly remark that there are no assumptions on $X$ besides that of satisfying (1.1) for some $\lambda \in \mathbb{R}$.

With the same reasoning as in the proof of Theorem 1.1 in [13], as a consequence of Lemma 2.1 and Proposition 2.3 we immediately deduce the validity of Theorem 1.1. As for Theorem 1.2 of [13], we state here the new improved version; again, its proof follows the same lines of [13] with the replacement of Lemma 3.3 there with the improved version in Proposition 2.3.

Theorem 2.4. Let $(M, g, X)$ be a complete, generic Ricci soliton of dimension $m \geq 3$, scalar curvature $S$, traceless Ricci tensor $T$ and Weyl tensor W. Suppose that

$$
\text { i) } S^{*}=\sup _{M} S(x)<+\infty ; \quad \text { ii) }|W|^{*}=\sup _{M}|W|<+\infty .
$$

Then, either $(M, g)$ is Einstein or $|T|^{*}=\sup _{M}|T|$ satisfies

$$
|T|^{*} \geq \frac{1}{2}\left(\sqrt{m(m-1)} \lambda-S^{*} \frac{m-2}{\sqrt{m(m-1)}}-\sqrt{\frac{m(m-2)}{2}}|W|^{*}\right) .
$$


In particular, if $(M, g)$ is conformally flat, then either $(M, g)$ has constant sectional curvature, or

$$
|T|^{*} \geq \frac{1}{2}\left(\sqrt{m(m-1)} \lambda-S^{*} \frac{m-2}{\sqrt{m(m-1)}}\right) .
$$

We now develop some further auxiliary results. First we recall the following formula due to Böchner, [23], and rediscovered many times in recent years.

Lemma 2.5. Let $Y$ be a vector field on the Riemannian manifold $(M, g)$. Then

$$
\operatorname{div}\left(\mathcal{L}_{Y} g\right)(Y)=\frac{1}{2} \Delta|Y|^{2}-|\nabla Y|^{2}+\operatorname{Ric}(Y, Y)+\nabla_{Y}(\operatorname{div} Y) .
$$

Remark 2.6. Formula (2.9) is a generalization of the usual Böchner formula. To see this let $u \in C^{3}(M)$ and let $Y=\nabla u$. Then, since $\frac{1}{2} \mathcal{L}_{\nabla u} g=\operatorname{Hess}(u)$, equation (2.9) becomes

$$
2 \operatorname{div}(\operatorname{Hess}(u))(\nabla u)=\frac{1}{2} \Delta|\nabla u|^{2}-|\operatorname{Hess}(u)|^{2}+\operatorname{Ric}(\nabla u, \nabla u)+g(\nabla \Delta u, \nabla u) .
$$

Since

$$
\operatorname{div}(\operatorname{Hess}(u))(\nabla u)=\operatorname{Ric}(\nabla u, \nabla u)+g(\nabla \Delta u, \nabla u),
$$

from the above we immediately deduce that (2.9) is, in this case, equivalent to

$$
\frac{1}{2} \Delta|\nabla u|^{2}=|\operatorname{Hess}(u)|^{2}+\operatorname{Ric}(\nabla u, \nabla u)+g(\nabla \Delta u, \nabla u),
$$

that is, the usual Böchner formula.

As a consequence of Lemma 2.5 we obtain the following (see also [18])

Proposition 2.7. Let $(M, g, X)$ be a generic Ricci soliton. Then

$$
\frac{1}{2} \Delta|X|^{2}=|\nabla X|^{2}-\operatorname{Ric}(X, X)
$$

or equivalently

$$
\frac{1}{2} \Delta_{X}|X|^{2}=|\nabla X|^{2}-\lambda|X|^{2}
$$

Proof. We trace the soliton equation (1.1) to obtain

$$
S+\operatorname{div} X=m \lambda
$$

and from this we deduce

$$
\nabla S=-\nabla \operatorname{div} X
$$

On the other hand, contracting twice the second Bianchi's identities we have the well-known formula

$$
\nabla S=2 \text { div Ric } .
$$

Thus, comparing (2.12) and (2.13), we obtain

$$
\nabla \operatorname{div} X=-2 \operatorname{div} \text { Ric . }
$$

Now taking the divergence of (1.1) and using the fact that $\operatorname{div}(\lambda g)=0$ we find

$$
\operatorname{div}\left(\mathcal{L}_{X} g\right)=-2 \operatorname{div} \text { Ric, }
$$

and from (2.14) we deduce

$$
\nabla \operatorname{div} X=\operatorname{div}\left(\mathcal{L}_{X} g\right)
$$

In particular

$$
\nabla_{X} \operatorname{div} X=\operatorname{div}\left(\mathcal{L}_{X} g\right)(X) .
$$


Substituting into (2.9) we immediately obtain (2.10). As for (2.11), using (2.10) and the definition of $\Delta_{X}$ given in (2.1), we have

$$
\frac{1}{2} \Delta_{X}|X|^{2}=|\nabla X|^{2}-\lambda|X|^{2}+\frac{1}{2}\left(\mathcal{L}_{X} g(X, X)-g\left(X, \nabla|X|^{2}\right)\right),
$$

from which (2.11) follows since

$$
\mathcal{L}_{X} g(X, X)=g\left(X, \nabla|X|^{2}\right)
$$

The following formulas can be verified by a simple direct computation. For any vector fields $Y, Z$ and functions $u, v \in C^{2}(M)$, with $v \neq 0$ on $M$,

$$
\begin{gathered}
\Delta_{Y}\left(\frac{u}{v}\right)=\frac{1}{v} \Delta_{Y} u-\frac{u}{v^{2}} \Delta_{Y} v-2 g\left(\nabla\left(\frac{u}{v}\right), \frac{\nabla v}{v}\right), \\
\Delta_{Y+Z} u=\Delta_{Y} u-g(Z, \nabla u) .
\end{gathered}
$$

The next computational result uses Proposition 2.7.

Lemma 2.8. Let $(M, g, X)$ be a generic Ricci soliton and $\alpha \in(0,1]$. Suppose $S>0$ on $M$. Then

$$
\Delta_{X-2 \nabla \log S}\left(\frac{|X|^{2}}{S^{\alpha}}\right) \leq \frac{2}{S^{\alpha}}\left\{\left(\frac{2-\alpha}{\alpha}\right)|\nabla X|^{2}-\left[(\alpha+1) \lambda-\alpha \frac{|\operatorname{Ric}|^{2}}{S}\right]|X|^{2}\right\} .
$$

Proof. Using equations (2.3) and (2.11), with the aid of (2.16) and (2.17), we compute

$$
\begin{aligned}
\Delta_{X-2 \nabla \log S}\left(\frac{|X|^{2}}{S^{\alpha}}\right)= & \frac{2}{S^{\alpha}}\left\{|\nabla X|^{2}-\left[(\alpha+1) \lambda-\alpha \frac{|\mathrm{Ric}|^{2}}{S}\right]|X|^{2}\right\} \\
& +\frac{2(1-\alpha)}{S^{\alpha+1}} g\left(\nabla|X|^{2}, \nabla S\right)+\frac{\alpha(\alpha-1)}{S^{\alpha+2}}|X|^{2}|\nabla S|^{2} .
\end{aligned}
$$

Next we use the inequality

$$
\left.|\nabla| X\right|^{2}|\leq 2| X|| \nabla X \mid
$$

and Cauchy and Young's inequalities with $\varepsilon>0$ to get

$$
\frac{1}{S^{\alpha+1}} g\left(\nabla S, \nabla|X|^{2}\right) \leq \frac{2|X||\nabla X||\nabla S|}{S^{\alpha+1}} \leq \frac{1}{\varepsilon} \frac{|\nabla X|^{2}}{S^{\alpha}}+\frac{\varepsilon}{S^{\alpha+2}}|X|^{2}|\nabla S|^{2} .
$$

Hence, for any $\alpha \in(0,1]$, inserting the previous inequalities into (2.19) and rearranging terms we obtain

$$
\begin{aligned}
\Delta_{X-2 \nabla \log S}\left(\frac{|X|^{2}}{S^{\alpha}}\right) \leq & \frac{2}{S^{\alpha}}\left\{\left[1+\frac{1-\alpha}{\varepsilon}\right]|\nabla X|^{2}-\left[(\alpha+1) \lambda-\alpha \frac{|\operatorname{Ric}|^{2}}{S}\right]|X|^{2}\right\} \\
& +\frac{1-\alpha}{S^{\alpha+2}}(2 \varepsilon-\alpha)|X|^{2}|\nabla S|^{2} .
\end{aligned}
$$

Choosing $\varepsilon=\frac{\alpha}{2}$ we finally have

$$
\Delta_{X-2 \nabla \log S}\left(\frac{|X|^{2}}{S^{\alpha}}\right) \leq \frac{2}{S^{\alpha}}\left\{\left(\frac{2-\alpha}{\alpha}\right)|\nabla X|^{2}-\left[(\alpha+1) \lambda-\alpha \frac{|\operatorname{Ric}|^{2}}{S}\right]|X|^{2}\right\} .
$$

that is, (2.18).

Corollary 2.9. Let $(M, g, X)$ be a generic, shrinking, complete Ricci soliton and assume that $S>0$, $S^{*}=\sup _{M} S<+\infty, \mid$ Ric $\mid \leq \Lambda S$ for some constant $\Lambda>0$ and

$$
|\nabla X|=o(|X|) \quad \text { as } r(x) \rightarrow+\infty .
$$

Then there exists $\alpha \in(0,1]$ and a compact $K=K_{\alpha} \subset M$ such that

$$
\Delta_{X-2 \nabla \log S}\left(\frac{|X|^{2}}{S^{\alpha}}\right)<0 \quad \text { on } M \backslash K .
$$

Proof. An immediate consequence of the assumptions and of (2.18). 
Our last ingredient for the proof of the main results comes from Lemma 2.1.

Lemma 2.10. Let $(M, g, X)$ be a generic Ricci soliton of dimension $m$ with scalar curvature $S>0$ on M. Then

$$
\begin{aligned}
\frac{1}{2} \Delta_{X-2 \nabla \log S}\left(\frac{|T|^{2}}{S^{2}}\right) \geq & 2 \frac{|T|^{2}}{S^{3}}\left(|T|-\frac{1}{\sqrt{m(m-1)}} S\right)^{2}+\frac{1}{S^{3}}\left(\frac{|T|}{\sqrt{S}}|\nabla S|-\sqrt{S}|\nabla T|\right)^{2} \\
& -\sqrt{\frac{2(m-2)}{m-1}} \frac{1}{S^{2}}|W||T|^{2}
\end{aligned}
$$

Proof. We use equations (2.16), (2.3) and (2.4) and

$$
|\operatorname{Ric}|^{2}=|T|^{2}+\frac{S^{2}}{m}
$$

to compute

$$
\Delta_{X-2 \nabla \log S}\left(\frac{|T|^{2}}{S^{2}}\right)=A+B+C,
$$

where

$$
\begin{aligned}
A & =\frac{2}{S^{3}}\left(S|\nabla T|^{2}+\frac{1}{S}|T|^{2}|\nabla S|^{2}-\left\langle\nabla|T|^{2}, \nabla S\right\rangle\right) \\
B & =\frac{8}{m-2} \frac{1}{S^{2}} \operatorname{tr}\left(t^{3}\right)+\frac{4}{m(m-1)} \frac{|T|^{2}}{S}+4 \frac{|T|^{4}}{S^{3}} \\
C & =-\frac{4}{S^{2}} T_{i k} T_{s j} W_{k s i j} .
\end{aligned}
$$

Next we use Cauchy inequality and

$$
\left.|\nabla| T\right|^{2}|\leq 2| \nabla T|| T \mid
$$

to obtain

$$
A \geq \frac{2}{S^{3}}\left(\frac{|T|}{\sqrt{S}}|\nabla S|-\sqrt{S}|\nabla T|\right)^{2} .
$$

Since $T$ is trace free, by Okumura's lemma, [15],

$$
\operatorname{tr}\left(t^{3}\right) \geq-\frac{m-2}{\sqrt{m(m-1)}}|T|^{3}
$$

with equality holding if and only if either $|T|=0$ or $|T|=\frac{1}{\sqrt{m(m-1)}} S$. Therefore

$$
B \geq 4 \frac{|T|^{2}}{S^{3}}\left(|T|-\frac{1}{\sqrt{m(m-1)}} S\right)^{2} .
$$

Finally, by Huisken's inequality, [11, Lemma 2.4],

$$
C \geq-\frac{2 \sqrt{2}}{S^{2}} \sqrt{\frac{m-2}{m-1}}|W||T|^{2} .
$$

Inequality (2.20) now follows immediately by putting together (2.21), (2.22), (2.23) and (2.24).

\section{Some AnAlytic Results}

Let $(M, g)$ be an $m$-dimensional Riemannian manifold, $X$ a vector field and $T$ a semipositive definite, symmetric $(0,2)$-tensor on $M$. We define the operator $L=L_{T, X}$ on $M$ acting on $u \in \operatorname{Lip}_{\text {loc }}(M)$ by

$$
L u=L_{T, X} u=\operatorname{div}\left(T(\nabla u,)^{\sharp}\right)-g(X, \nabla u),
$$

where of course the above has to be understood in the weak sense. We introduce the following 
Definition 3.1. i) We say that $M$ is $L$-parabolic if each bounded above, L-subharmonic function is constant, that is, each $u \in \operatorname{Lip}_{\text {loc }}(M)$ with $u^{*}=\sup _{M} u<+\infty$ and satisfying

$$
L u \geq 0 \quad \text { on } M
$$

is constant.

ii) We say that $M$ is strongly $L$-parabolic if for each non-constant $u \in \operatorname{Lip}_{\text {loc }}(M)$ with $u^{*}<\infty$ and for each $\eta>0$

$$
\inf _{\Omega_{\eta}} L u<0
$$

where

$$
\Omega_{\eta}=\left\{x \in M: u(x)>u^{*}-\eta\right\} .
$$

Of course, again, (3.2) has to be interpreted in the weak sense, that is, for some $\varepsilon>0$ there exists $\varphi \in \operatorname{Lip}_{0}\left(\Omega_{\eta}\right), \varphi \geq 0, \varphi \neq 0$, such that

$$
-\left(\int_{\Omega_{\eta}} T(\nabla u, \nabla \varphi)+g(X, \nabla u) \varphi\right) \leq-\int_{\Omega_{\eta}} \varepsilon \varphi .
$$

It is clear that strong parabolicity implies parabolicity, and the converse can be shown to be true whenever the function obtained as the maximum between a constant and a $L$-subharmonic function is still $L$-subharmonic. This is the case for the operators in (3.1), as we are going to prove by adapting an idea of Agmon, [1].

Proposition 3.2. Let $L$ be the operator defined in(3.1); let $u \in$ Lip $_{\text {loc }}(M)$ satisfy

$$
L u \geq 0 \quad \text { on } M
$$

and let $\alpha \in \mathbb{R}$ be any constant. Then the function defined by

$$
w(x)=\max \{u(x), \alpha\}
$$

satisfies (3.3).

Proof. Since $u+\beta$ is still a solution of (3.3) for every $\beta \in \mathbb{R}$, without loss of generality we can suppose that $\alpha=0$. In this case $w=u^{+}$, so that it remains to show that $u^{+}$is a solution of (3.3). Towards this end we recall that (3.3) means

$$
\int_{M} T(\nabla u, \nabla \varphi)+g(X, \nabla u) \varphi \leq 0
$$

for every $\varphi \in \operatorname{Lip}_{0}(M)$ such that $\varphi \geq 0$. We fix such a $\varphi$ and a constant $\varepsilon>0$. We set

$$
u_{\varepsilon}=\sqrt{u^{2}+\varepsilon^{2}}, \quad \varphi_{\varepsilon}=\frac{1}{2}\left(1+\frac{u}{u_{\varepsilon}}\right) \varphi
$$

and note that $\varphi_{\varepsilon}$ is still an admissible test function for (3.3). Furthermore, $u_{\varepsilon}>|u|$ and

$$
u_{\varepsilon} \rightarrow|u|, \quad \nabla u_{\varepsilon} \rightarrow \nabla|u|, \quad \varphi_{\varepsilon} \rightarrow\left(\frac{1+\operatorname{sgn}(u)}{2}\right) \varphi \quad \text { as } \varepsilon \rightarrow 0^{+} .
$$

A simple computation shows that

$$
T\left(\nabla u_{\varepsilon}, \nabla \varphi\right)=T\left(\nabla u, \nabla\left(\frac{u}{u_{\varepsilon}} \varphi\right)\right)-\frac{\varphi}{u_{\varepsilon}^{3}}\left(u_{\varepsilon}^{2}-u^{2}\right) T(\nabla u, \nabla u)
$$

so that, since $T$ is, a fortiori, semipositive definite, we obtain

$$
T\left(\nabla u_{\varepsilon}, \nabla \varphi\right) \leq T\left(\nabla u, \nabla\left(\frac{u}{u_{\varepsilon}} \varphi\right)\right) .
$$

From this inequality it immediately follows

$$
T\left(\nabla\left(\frac{u+u_{\varepsilon}}{2}\right), \nabla \varphi\right) \leq T\left(\nabla u, \nabla \varphi_{\varepsilon}\right) .
$$


Now by the definition of subsolution

$$
\int_{M} T\left(\nabla u, \nabla \varphi_{\varepsilon}\right)+g(X, \nabla u) \varphi_{\varepsilon} \leq 0
$$

and therefore, using (3.5),

$$
\int_{M} T\left(\nabla\left(\frac{u+u_{\varepsilon}}{2}\right), \nabla \varphi\right)+g(X, \nabla u) \varphi_{\varepsilon} \leq 0 .
$$

Letting $\varepsilon \downarrow 0^{+}$we deduce by (3.4) and by Fatou's lemma that

$$
\int_{M} T\left(\nabla u^{+}, \nabla \varphi\right)+g\left(X, \nabla u^{+}\right) \varphi \leq 0,
$$

that is, $u^{+}$is a subsolution of (3.3).

The following is a sufficient condition for the validity of strong $L$-parabolicity.

Theorem 3.3. Let $(M, g)$ be a connected Riemannian manifold and $L$ be as in (3.1). Let $\gamma \in \operatorname{Lip}_{\text {loc }}(M)$ satisfy

$$
\begin{aligned}
& \gamma(x) \rightarrow+\infty \text { as } x \rightarrow \infty \\
& L \gamma<0 \text { outside a compact set. }
\end{aligned}
$$

Then $M$ is $L$-parabolic.

Proof. We reason by contradiction and we assume the existence of a non-constant $u \in \operatorname{Lip}_{l o c}(M)$, with $u^{*}<+\infty$ and of $\eta>0$ such that

$$
L u \geq 0 \quad \text { on } \Omega_{\eta} .
$$

First we observe that $u^{*}$ cannot be attained at any point $x_{0} \in M$, for otherwise $x_{0} \in \Omega_{\eta}$ and by the strong maximum principle for the operator $L$ given in Theorem 5.6 of [20] we have that $u$ is constantly equal to $u^{*}$ on the connected component of $\Omega_{\eta}$ containing $x_{0}$. From this and connectedness it follows easily that $u$ is constant on $M$, a contradiction.

Next for $t \in \mathbb{R}$ we let

$$
\Lambda_{t}=\{x \in M: \gamma(x)>t\}
$$

and we define

$$
u_{t}^{*}=\sup _{x \in \Lambda_{t}^{c}} u(x) .
$$

Using (3.6) it is not hard to see that $\Lambda_{t}^{c}$ is compact, and therefore $u_{t}^{*}$ is attained at some point of $\Lambda_{t}^{c}$. Since $u^{*}$ is not attained in $M$ and $\left\{\Lambda_{t}^{c}\right\}$ is a nested family of compact sets exhausting $M$, there exists a diverging sequence $\left\{t_{j}\right\}_{j \in \mathbb{N}} \subset \mathbb{R}^{+}$such that

$$
u_{t_{j}}^{*} \nearrow u^{*} \text { as } j \rightarrow+\infty,
$$

and we can choose $T_{1}>0$ sufficiently large so that

$$
u_{T_{1}}^{*}>u^{*}-\frac{\eta}{2}
$$

Without loss of generality we can also suppose to have chosen $T_{1}$ large enough so that also (3.7) holds on $\Lambda_{T_{1}}$. Now fix $\alpha$ satisfying $u_{T_{1}}^{*}<\alpha<u^{*}$. Because of (3.9) we can find $j \in \mathbb{N}$ sufficiently large so that

$$
T_{2}=t_{j}>T_{1} \quad \text { and } \quad u_{T_{2}}^{*}>\alpha
$$

We select $\bar{\eta}>0$ small enough to satisfy

$$
\alpha+\bar{\eta}<u_{T_{2}}^{*}
$$

For $\sigma>0$ we define

$$
\gamma_{\sigma}(x)=\alpha+\sigma\left(\gamma(x)-T_{1}\right)
$$


Then

$$
L \gamma_{\sigma}=\sigma L \gamma<0 \quad \text { on } \Lambda_{T_{1}} \text {. }
$$

Next we observe that

$$
\alpha \leq \gamma_{\sigma}(x) \leq \alpha+\sigma\left(T_{2}-T_{1}\right) \quad \text { on } \Lambda_{T_{1}} \backslash \Lambda_{T_{2}}
$$

and therefore we can choose $\sigma>0$ sufficiently small to guarantee

$$
\sigma\left(T_{2}-T_{1}\right)<\bar{\eta}
$$

and then

$$
\alpha \leq \gamma_{\sigma}(x)<\alpha+\bar{\eta} \quad \text { on } \Lambda_{T_{1}} \backslash \Lambda_{T_{2}} .
$$

On $\partial \Lambda_{T_{1}}$ we have

$$
\gamma_{\sigma}(x)=\alpha>u_{T_{1}}^{*} \geq u(x)
$$

so that

$$
u(x)-\gamma_{\sigma}(x)<0 \quad \text { and } \partial \Lambda_{T_{1}} .
$$

Furthermore, if $\bar{x} \in \Lambda_{T_{1}} \backslash \Lambda_{T_{2}}$ is such that

$$
u(\bar{x})=u_{T_{2}}^{*}>\alpha+\bar{\eta}
$$

then

$$
u(\bar{x})-\gamma_{\sigma}(\bar{x}) \geq u_{T_{2}}^{*}-\alpha-\sigma\left(T_{2}-T_{1}\right)>u_{T_{2}}^{*}-\alpha-\bar{\eta}>0
$$

because of (3.10) and (3.12). Finally (3.6) and the fact that $u^{*}<+\infty$ imply

$$
\left(u-\gamma_{\sigma}\right)(x)<0 \quad \text { on } \Lambda_{T_{3}}
$$

for $T_{3}>T_{2}$ sufficiently large. Hence

$$
\mu=\sup _{x \in \bar{\Lambda}_{T_{1}}}\left(u-\gamma_{\sigma}\right)(x)>0
$$

and it is in fact a positive maximum attained at some point $z_{0}$ in the compact set $\bar{\Lambda}_{T_{1}} \backslash \Lambda_{T_{3}}$. Thus

$$
\Sigma=\left\{x \in \Lambda_{T_{1}}:\left(u-\gamma_{\sigma}\right)(x)=\mu\right\} \neq \emptyset .
$$

Furthermore, for $y \in \Sigma$,

$$
u(y)=\gamma_{\sigma}(y)+\mu>\gamma_{\sigma}(y)=\alpha+\sigma\left(\gamma(y)-T_{1}\right)>\alpha>u_{T_{1}}^{*}>u^{*}-\frac{\eta}{2},
$$

so that

$$
\Sigma \subset \Omega_{\eta},
$$

and by (3.13) $\Sigma \subset \bar{\Lambda}_{T_{1}} \backslash \Lambda_{T_{3}}$, therefore $\Sigma$ is compact. Hence there exists an open neighbourhood of $\Sigma$, $\Sigma_{U} \subset \Omega_{\eta}$. Fix $y \in \Sigma$ and $\beta \in(0, \mu)$ and call $\Sigma_{\beta, y}$ the connected component of the set

$$
\left\{x \in \Lambda_{T_{1}}:\left(u-\gamma_{\sigma}\right)(x)>\beta\right\}
$$

containing $y$. We can choose $\beta$ sufficiently close to $\mu$ so that $\bar{\Sigma}_{\beta, y} \subset \Omega_{\eta} \cap \Lambda_{T_{1}}$. Note that, since $\beta>0$, $\bar{\Sigma}_{\beta, y}$ is compact. Because of (3.11) and (3.8)

$$
L u \geq 0 \geq \sigma L \gamma=L \gamma_{\sigma} \quad \text { on } \Sigma_{\beta, y}
$$

in the weak sense. Furthermore, $u(x)=\gamma_{\sigma}(x)+\beta$ on $\partial \Sigma_{\beta, y}$. By Theorem 5.3 of $[20], u(x) \leq \gamma_{\sigma}(x)+\beta$ on $\bar{\Sigma}_{\beta, y}$. However $y \in \Sigma_{\beta, y}$ and we have

$$
u(y)=\gamma_{\sigma}(y)+\mu>\gamma_{\sigma}(y)+\beta
$$

by our choice of $\beta$. Contradiction. 
We remark that for (3.13) to hold it is enough to require

$$
u(x)=o(\gamma(x)) \quad \text { as } x \rightarrow \infty .
$$

Hence a careful reading of the above proof yields the validity of the following Liouville-type result.

Theorem 3.4. Let $(M, g)$ be a connected Riemannian manifold and L as in (3.1). Let $\gamma \in \operatorname{Lip}_{\text {loc }}(M)$ satisfy (3.6) and (3.7). If $u \in$ Lip $_{\text {loc }}(M)$ satisfies (3.14) and Lu $\geq 0$ on $M$ then $u$ is constant.

\section{Proof of Theorems 1.2 And 1.3}

In this short section we prove Theorem 1.2 and Theorem 1.3. First of all, from Theorem 1.1 we know that the soliton is either flat or it has positive scalar curvature $S>0$. Moreover, from the growth estimates on the vector field $X$ proved in [14, Remark 2.2], we know that $|X| \rightarrow \infty$ as $r \rightarrow \infty$ (we notice that, under the assumptions of theorems 1.2 and 1.3, one has that the metric has bounded Ricci curvature).

In dimension three, every complete shrinking soliton has nonnegative sectional curvature [7]. Moreover, by Hamilton's strong maximum principle, either $g$ has strictly positive sectional curvature or it splits a line. In this latter case, either the soliton is flat or it is isometric to a quotient of the round cylinder $\mathbb{R} \times \mathbb{S}^{2}$. So from now on, in dimension three, we can assume that the metric has strictly positive sectional curvature. In particular it holds $|\mathrm{Ric}|^{2}<\frac{1}{2} S^{2}$. Moreover, the pinching condition (1.5) is automatically satisfied, since the W.eyl tensor vanishes in three dimension. Thus, it is sufficient to prove Theorem 1.3 , with $m \geq 3$, to conclude. Now, the proof follows the arguments in [5]. Under the assumptions of Theorem 1.3, Corollary 2.9 applies. Hence, from Lemma 2.10 and Theorem 3.3, we have that $\frac{|T|^{2}}{S^{2}}$ must be constant on $M$. Moreover, from the proof of Lemma 2.10, we get that $(M, g)$ is either Einstein or satisfies the identity $|T|=\frac{1}{\sqrt{m(m-1)}} S$. Now, if $m=3$, this violates the fact that the metric has positive sectional curvature. So $(M, g)$ is Einstein, hence it has constant positive sectional curvature and is a finite quotient of $\mathbb{S}^{3}$. On the other hand, if $m \geq 4$, the pinching assumption (1.5) on the Weyl curvature implies that $(M, g)$ is either Einstein or has zero Weyl tensor. In the first case, since the metric has positive scalar curvature, we have that $M$ is compact. Moreover, from the pinching condition (1.5), we get that

$$
|W|^{2} \leq \frac{2}{m^{2}(m-1)(m-2)} S^{2} \leq \frac{4}{m(m-1)(m-2)(m+1)} S^{2}
$$

Thus, the pinching condition in Huisken [11, Corallary 2.5] is satisfied which implies that $(M, g)$ has positive curvature. Hence, from a classical theorem of Tachibana [21], we conclude that $(M, g)$ has constant positive sectional curvature and is a finite quotient of $\mathbb{S}^{m}$. On the other hand, if the Weyl tensor vanishes, from the classification of locally conformally flat shrinking Ricci solitons given in [6] we obtain that if $(M, g)$ is non-flat and noncompact, then it must be a finite quotient of $\mathbb{R} \times \mathbb{S}^{m-1}$.

This concludes the proof of Theorem 1.2 and Theorem 1.3.

\section{FURTHER REMARKS}

In this final section we collect some further observations on generic Ricci solitons. We begin with the following

Lemma 5.1. Let $Y$ be a vector field on $(M, g), S$ the scalar curvature, $T=$ Ric $-\frac{S}{m} g$ the traceless Ricci tensor with corresponding endomorphism $t: T M \rightarrow T M$ and $l_{Y} g: T M \rightarrow T M$ the endomorphism corresponding to $\mathcal{L}_{Y} g$, that is, for every vector field $Z$

$$
\left(l_{Y} g\right)(Z)=\left(\mathcal{L}_{Y} g(Z)\right)^{\sharp} .
$$


We set $W_{Y}=T(Y,)^{\sharp}=t(Y)$. Then

$$
\operatorname{div} W_{Y}=\frac{1}{2} \operatorname{tr}\left(l_{Y} g \circ t\right)+\frac{m-2}{2 m} Y(S) .
$$

Proof. We give the short proof for completeness. In the moving frame notation $W_{Y}$ is the vector field of components

$$
W_{j}=\left(W_{Y}\right)_{j}=Y_{i} T_{i j}
$$

Thus

$$
\operatorname{div} W_{Y}=Y_{i k} T_{i k}+Y_{i} T_{i k, k} .
$$

Using the fact that $T$ is symmetric and Schur's identity

$$
2 R_{i k, i}=S_{k}
$$

we have

$$
\operatorname{div} W_{Y}=\frac{1}{2}\left(Y_{i k}+Y_{k i}\right) T_{i k}+Y_{i}\left(R_{i k, k}-\frac{S_{i}}{m}\right)=\frac{1}{2} \operatorname{tr}\left(l_{Y} g \circ t\right)+\frac{m-2}{2 m} S_{i} Y_{i}
$$

that is, (5.1).

As a consequence we obtain

Proposition 5.2. Let $(M, g, X)$ be a generic Ricci soliton with scalar curvature $S$ and traceless Ricci tensor $T$. Then

$$
\operatorname{div}\left(T(X,)^{\sharp}\right)=\frac{m-2}{2 m} X(S)-|T|^{2} .
$$

Proof. Use the soliton equation (1.1), the definition of $T$ and $|T|^{2}=\mid$ Ric $\left.\right|^{2}-\frac{S^{2}}{m}$ into (5.1) to obtain (5.2).

Remark 5.3. Note that (5.2) could be interpreted as a kind of infinitesimal "Kazdan-Warner" condition for generic Ricci solitons.

The following result is an immediate consequence of Proposition 5.2.

Theorem 5.4. Let $(M, g, X)$ be a complete, generic Ricci soliton with constant scalar curvature and dimension $m \geq 3$. Let $T$ be the trace free Ricci tensor and assume that for $p, q$ conjugate exponents

$$
|X| \in L^{p}(M), \quad|T| \in L^{q}(M) .
$$

Then $(M, g)$ is Einstein, $X$ is either Killing or homothetic (but not Killing). In this latter case $(M, g, X)$ is not steady and $(M, g)$ is locally Euclidean.

Proof. Since $S$ is constant, (5.2) becomes

$$
\operatorname{div}\left(T(X,)^{\sharp}\right)=-|T|^{2} \leq 0 .
$$

Furthermore, because of (5.3) the vector field $T(X,)^{\sharp} \in L^{1}(M)$. We apply Karp's version of the divergence theorem [12] to deduce $\int_{M}|T|^{2}=0$, that is, $(M, g)$ is Einstein. From the soliton equation $(1.1)$

$$
\frac{1}{2} \mathcal{L}_{X} g=\left(\lambda-\frac{S}{m}\right) g .
$$

If $X$ is not Killing $\lambda-\frac{S}{m} \neq 0$, and by Theorem 4.1 in [22], $(M, g)$ is locally Euclidean so that $S \equiv 0$ and $\lambda \neq 0$.

\section{REFERENCES}

[1] S. Agmon. Bounds on exponential decay of eigenfunctions of Schrödinger operators. In Schrödinger operators (Como, 1984), volume 1159 of Lecture Notes in Math., pages 1-38. Springer, Berlin, 1985.

[2] L. J. Alías, M. Dajczer, and M. Rigoli. Higher order mean curvature estimates for bounded complete hypersurfaces. Nonlinear Anal., 84:73-83, 2013. 
[3] H.-D. Cao, B.-L. Chen, and X.-P. Zhu. Recent developments on Hamilton's Ricci flow. In Surveys in differential geometry. Vol. XII. Geometric flows, volume 12 of Surv. Differ. Geom., pages 47-112. Int. Press, Somerville, MA, 2008.

[4] H.-D. Cao and D. Zhou. On complete gradient shrinking Ricci solitons. J. Differential Geom., 85(2):175-185, 2010.

[5] G. Catino. Complete gradient shrinking Ricci solitons with pinched curvature. Math. Ann., 355(2):629-635, 2013.

[6] G. Catino, C. Mantegazza, and L. Mazzieri. Locally conformally flat ancient ricci flows. arXiv:1308.2374v4 [math.DG], 2013.

[7] B.-L. Chen. Strong uniqueness of the Ricci flow. J. Differential Geom., 82(2):363-382, 2009.

[8] S. Y. Cheng and S. T. Yau. Differential equations on Riemannian manifolds and their geometric applications. Comm. Pure Appl. Math., 28(3):333-354, 1975.

[9] M. Fernández-López and E. García-Río. Maximum principles and gradient Ricci solitons. J. Differential Equations, 251(1):73-81, 2011.

[10] R. S. Hamilton. The formation of singularities in the Ricci flow. In Surveys in differential geometry, Vol. II (Cambridge, MA, 1993), pages 7-136. Int. Press, Cambridge, MA, 1995.

[11] G. Huisken. Ricci deformation of the metric on a Riemannian manifold. J. Differential Geom., 21(1):47-62, 1985.

[12] L. Karp. On Stokes' theorem for noncompact manifolds. Proc. Amer. Math. Soc., 82(3):487-490, 1981.

[13] P. Mastrolia, M. Rigoli, and M. Rimoldi. Some geometric analysis on generic Ricci solitons. Commun. Contemp. Math., 15(3):1250058, 25, 2013.

[14] A. Naber. Noncompact shrinking four solitons with nonnegative curvature. J. Reine Angew. Math., 645:125-153, 2010.

[15] M. Okumura. Hypersurfaces and a pinching problem on the second fundamental tensor. Amer. J. Math., 96:207-213, 1974.

[16] G. Perelman. The entropy formula for the Ricci flow and its geometric applications. arXiv:math/0211159v1 [math.DG], 2002.

[17] G. Perelman. Ricci flow with surgery on three manifolds. arXiv:math/0303109v1 [math.DG], 2003.

[18] P. Petersen and W. Wylie. Rigidity of gradient Ricci solitons. Pacific J. Math., 241(2):329-345, 2009.

[19] S. Pigola, M. Rigoli, and A. G. Setti. Vanishing and finiteness results in geometric analysis, volume 266 of Progress in Mathematics. Birkhäuser Verlag, Basel, 2008. A generalization of the Bochner technique.

[20] P. Pucci, M. Rigoli, and J. Serrin. Qualitative properties for solutions of singular elliptic inequalities on complete manifolds. J. Differential Equations, 234(2):507-543, 2007.

[21] S. Tachibana. A theorem of Riemannian manifolds of positive curvature operator. Proc. Japan Acad., 50:301-302, 1974.

[22] Y. Tashiro. Complete Riemannian manifolds and some vector fields. Trans. Amer. Math. Soc., 117:251-275, 1965.

[23] K. Yano and S. Bochner. Curvature and Betti numbers. Annals of Mathematics Studies, No. 32. Princeton University Press, Princeton, N. J., 1953. 